\title{
Adults imitate to send a social signal
}

Sujatha Krishnan-Barman and Antonia F de C Hamilton

Institute of Cognitive Neuroscience, University College London

Alexandra House, 17 Queen Square

London WC1N 3AR

United Kingdom

Email: sujatha.krishnan-barman.14@ucl.ac.uk

a.hamilton@ucl.ac.uk

Corresponding Author:

Antonia Hamilton

a.hamilton@ucl.ac.uk

Running head: Imitation as a social signal

Word count: 2,998 words

Preprint of a paper accepted in Cognition, 2019

https://www.sciencedirect.com/science/article/pii/S0010027719300629 


\begin{abstract}
Humans frequently imitate each other's actions with high fidelity, and different reasons have been proposed for why they do so. Here we test the hypothesis that imitation can act as a social signal, with imitation occurring with greater fidelity when a participant is being watched. In a preregistered study, 30 pairs of naïve participants played an augmentedreality game involving moving blocks in space. We compared imitation fidelity between trials where the leader watched the followers' action, and trials where the leader did not watch. Followers imitated the trajectory height demonstrated by the leader, and critically, the strength of this correlation was greater in trials where the follower knew the leader was watching them. This suggests that followers spontaneously used imitation as a social signal in a nonverbal interaction task, supporting socio-communicative hypotheses of imitation.
\end{abstract}

Keywords: Imitation, audience effect, social signalling, dyadic interaction 


\section{Introduction}

Humans imitate prolifically, from early childhood through to adulthood, and even when imitation is not strictly necessary (Nadel, 2002; Whiten et al., 2016), but we are yet to fully understand why. A variety of explanations have been advanced to explain imitation, including as a mechanism to learn new skills (Flynn \& Smith, 2012), as a by-product of domain-general learning (Heyes, 2017), or as a way to boost social affiliation (Over \& Carpenter, 2013; Uzgiris, 1981). This latter theory, also known as the 'social glue hypothesis' (Lakin, Jefferis, Cheng, \& Chartrand, 2003), suggests that imitation is a social signal which can influence an interaction (Wang \& Hamilton, 2012). The aim of the current paper is to test this social-signalling hypothesis of imitation, in a robust and ecologically valid fashion.

The genesis of this idea comes from examining how it is possible for imitation to create affiliations between people. Such affiliation could emerge as a lucky side-effect of imitation, but the STORM model (Wang \& Hamilton, 2012) makes the more specific claim that imitation is performed in order to affiliate (Farmer, Ciaunica, \& Hamilton, 2018). We illustrate this with a scenario in which Alice imitates an action performed by Ben. If imitation influences affiliation, Ben should receive the signal 'I am imitating you' and change his attitude or behaviour towards Alice in response. This is supported by evidence that being imitated leads to an increase in liking (Lakin \& Chartrand, 2003). Further, if Alice imitates in order to send a signal to Ben, she should imitate him with greater fidelity when she knows he is watching her, compared with a time when she knows he is not watching her. Here we consider this latter prediction - that imitation should be produced with greater fidelity when the interaction partner is watching, and can therefore receive the social signal being transmitted.

Previous work testing if imitation increases when a participant is being watched (and can send a social signal) has yielded mixed results. Using video stimuli, Wang \& Hamilton (2011; 2013b) showed that imitation is enhanced when a direct gaze cue is present at the time of responding. A study of facial mimicry ${ }^{1}$ found stronger imitation of a wince following eye contact, supporting the social-signalling hypothesis (Bavelas, Black, Lemery, \& Mullett, 1986). In some studies, children imitate the irrelevant actions performed by a demonstrator

${ }^{1}$ Mimicry is a subset of imitation referring to copying of actions that are not goal directed. Overimitation, meanwhile, involves copying unnecessary or causally-irrelevant features of a goal-directed action (Hamilton, 2015). In this paper we use the neutral term imitation to refer to all copying behaviour, whether explicitly goal-directed or not. 
only when the demonstrator is present during the child's turn (Diyanni, Nini, \& Rheel, 2011; Nielsen \& Blank, 2011). However, other studies suggest that children overimitate even when the demonstrator is absent (Lyons, Young, \& Keil, 2007), and that both children and adults overimitate when they are not aware of being watched (Whiten et al., 2016). These latter studies argue against imitative behaviour serving as a social signal. However, these may be due to other confounding factors, which overshadow the social-signalling effect. Several of these studies use puzzle-box tasks where learning about a novel object may dominate the response. Many studies use confederates to demonstrate the to-be-imitated actions, which could lead to an experimenter effect (Gilder \& Heerey, 2018; Kuhlen \& Brennan, 2013). Other studies use video stimuli where participants know they are not really being watched, compromising ecological validity (Risko, Laidlaw, Freeth, Foulsham, \& Kingstone, 2012). Finally, the situations where someone is being watched versus one in which no one is watching can engender several possible cognitive changes (Bond, 1982), including social-facilitation effects, changes in anxiety due to direct gaze and changes in attention. One recent study has suggested that several results showing modulation of imitation by social context arise due to effects of attention or anxiety and are not related to social-signalling (Heyes, 2017). Given that in several extant studies the social context differs significantly between the watched condition and the unwatched condition, it has not so far been possible to explicitly test whether social signalling drives imitative behaviour in adults.
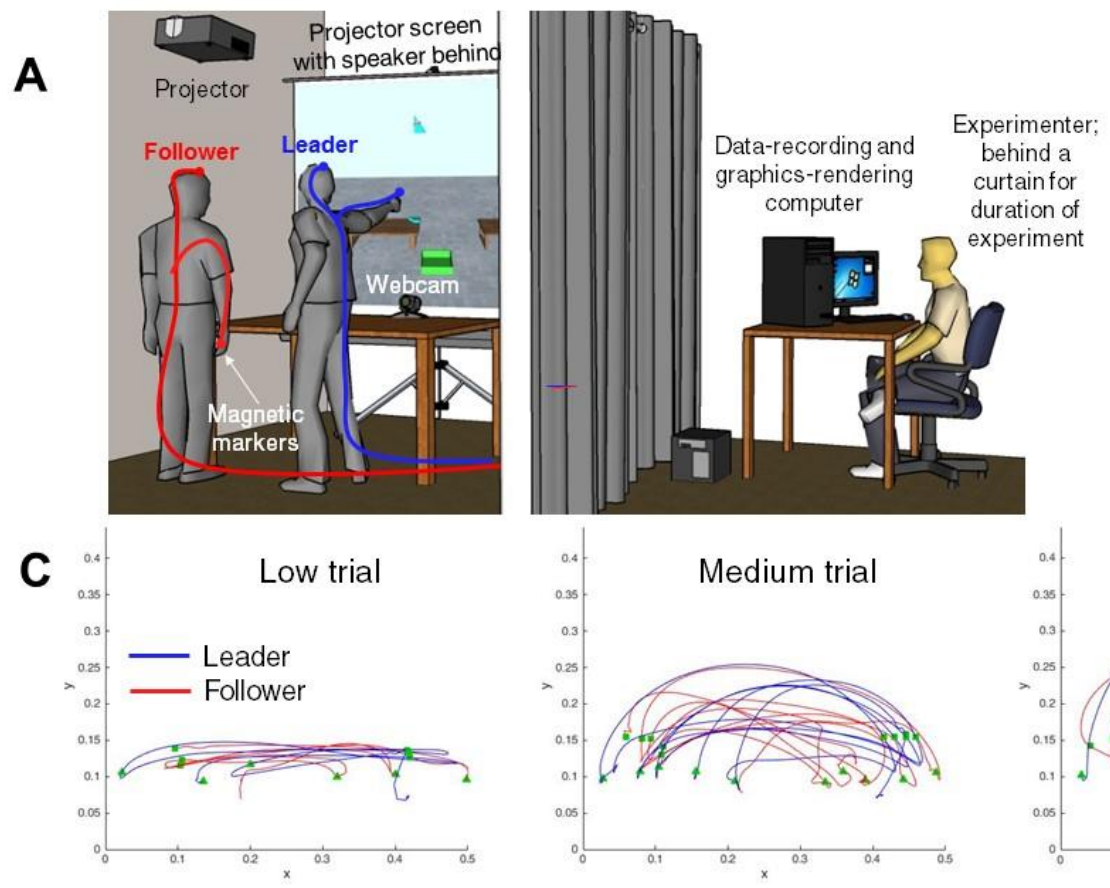

B
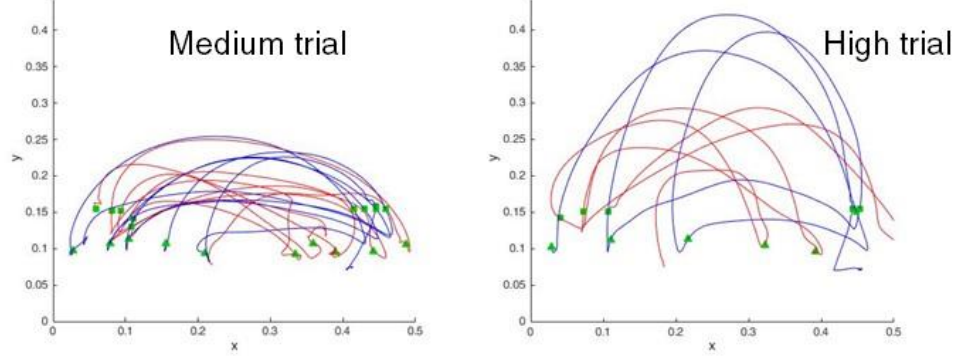

Figure 1. A. configuration of the augmented reality lab. Two participants standing sideby-side can see the AR space and are motion tracked. A curtain separated the participants 
from the experimenter. B. The task was to move blocks from one table to another in a demonstrated order. C. Sample trajectories from leader (blue) and follower (red) for trials with different demonstration heights.

The current study aims to test the social-signalling hypothesis of imitation in a rigorous manner, avoiding confounding factors that have affected previous studies. In this study pairs of naïve adult participants were asked to move blocks from one location to another in a specified order (Oliver, Tachtsidis, \& Hamilton, 2017) (Figure 1). This augmented-reality setup provided a rich interactive context while avoiding experimenter effects. Two independent variables were manipulated: the height of trajectory demonstrated by the Leader, and whether the Leader's eyes were open or closed during the Follower's turn. As the two participants stand side by side throughout there are no changes in eye contact or social facilitation between the two conditions. This is akin to studies of visual perspective taking using 'goggles' (Teufel, Fletcher, \& Davis, 2010) which have been accepted as a definitive test of 'social' information processing (Heyes, 2015). Finally, we resolve issues of variance in participant performance by using a simple task with clear rules for excluding non-compliant participants. The study was pre-registered to support a rigorous analysis scheme. This paper presents the results of the pre-registered study, based closely on an exploratory study (see Supplementary Materials).

Based on the STORM model, we predict that (a) participants will imitate the causally-irrelevant kinematic features of their partner's actions without being explicitly instructed to do so, and (b) participants will imitate to a greater extent when they know they are being watched by their partner, compared with a situation where they know their partner cannot see them.

\section{Materials and Methods}

\subsection{Participants}

The pre-registered study is a replication of an exploratory study (see Supplementary Material and https://osf.io/ezj8g/). A power-analysis in $\mathrm{G}^{*}$ Power (Faul, Erdfelder, Lang, \& Buchner, 2007) showed that 29 dyads would be sufficient to detect an effect of being watched with a power of 0.9. We therefore planned to collect data from 30 valid dyads. Dyads were excluded for failing pre-analysis quality checks (see Supplementary Material) and a total of 80 participants were tested in 40 pairs to collect data from 30 valid dyads (42 females, 18 males; mean age $=24.17$ years; $S D=6.58$ years). All were right-handed, had normal or corrected-to-normal vision and hearing, had no history of neurological or 
psychiatric disorders, and had not previously participated in this experiment. All procedures were approved by the UCL Research Ethics Committee.

\subsection{Procedure}

Pairs of participants arrived at the same time, and were asked to introduce themselves to each other and choose a 'team name' together; they were told they would be competing against other teams who had previously participated in the experiment. This was done to introduce a prosocial collaborative mind-set during the task. One participant was assigned the role of Leader and the other of the Follower; they stood side-by-side facing the screen (Leader/Follower locations were counterbalanced). Magnetic motion-trackers (Polhemus Liberty, Colchester Vermont) were fixed to the right hand and forehead of each participant. The hand markers allowed participants to control a hand icon in the augmentedreality environment and move objects (akin to a 3D mouse-pointer) (Fig 1A). Participants were instructed to move blocks from one table to another in a specified order (Fig 1B). The augmented-reality environment and experimental sequence were implemented in Vizard (WorldViz, Santa Barbara, CA).

The study had three phases: familiarisation, full trials and final trials. In the familiarisation phase, participants practiced moving blocks in the augmented-reality environment. At the start of each trial a (computerised) voice command instructed the Follower to close his/her eyes. The Leader then saw a demonstration of 3 or 4 blocks being moved in a specific order from one table to another (Fig 2A). Then the Follower heard a voice command to open their eyes, and the Leader demonstrated the block-movement task to the Follower (Fig 2B). Finally, the Follower was asked to move the blocks in the same order to the final table (Fig 2C). Both participants then saw a joint score based on accuracy (moving blocks in the right order) and timing (moving quickly) (Fig 2D). 


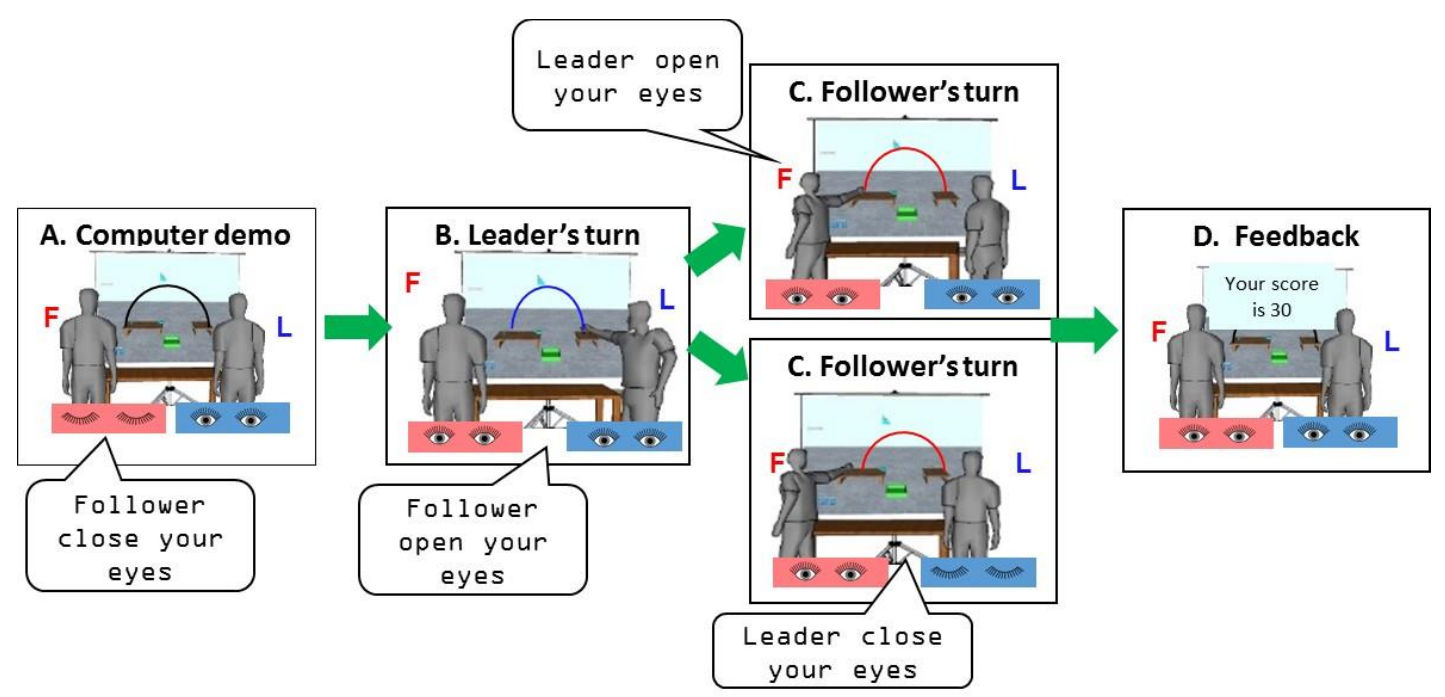

Figure 2. Trial timeline. A. The Follower closes their eyes while the Leader watches the computer demonstration. B. The Leader demonstrates, while the Follower watches. C. The Follower moves the blocks (The Leader's eyes can be open or closed). D. Participants see a joint score which rewards accuracy and speed. Speech bubbles throughout show computerised voice commands.

After three familiarisation trials, the experimental phase started. On the first trial, after the Follower closed their eyes, the Leader read an on-screen message with an additional 'secret' instruction to explicitly follow the trajectory demonstrated by the computer ${ }^{2}$. The computer demonstration then showed the blocks moving using a low, medium, or high trajectory, and the Leader was instructed to copy both the block order and the trajectory when demonstrating to the Follower. Leaders who failed to follow these instructions or shared this secret information with the Follower were excluded (see Supplementary Materials). On half the trials, prior to the Follower's turn the Leader was instructed to close their eyes. On the other half the Leader was instructed to keep their eyes open. Thus the ability of the Leader to monitor the Follower's movement was manipulated. All pairs completed 18 experimental trials (with three movement heights and the watched/unwatched conditions, each repeated thrice). The Leader's eyes were open or closed in blocks of three trials (with the order of watched blocks vs unwatched blocks

2 The exact wording of the instruction was as follows: "You should follow the same PATH as the demonstration. This means you should move the pieces along the same path (reaching the same height) as the demonstration! However, you should NOT share this instruction with your teammate". 
counterbalanced across pairs). Participants then completed the final phase of six trials where both the Leader and Follower were explicitly told to follow the trajectory to enable us to check that they understood this idea. The phases and conditions are summarised in Supplementary Table 1.

Finally, both participants separately completed a series of questionnaires and an open-ended debrief (see Supplementary Materials) to allow for exploratory analyses.

\subsection{Data analysis}

As specified in the pre-registration our primary analysis focused on a single parameter: the maximum height reached by each participant in each full trial. Since there were multiple pieces moved in each trial ( 3 or 4 depending on the trial), we believe peak height is the most salient measure of whether a movement trajectory was copied. The correlation coefficient $(\mathrm{R})$ between the maximum heights reached by the Leader and the Follower in the Watched trials and the Unwatched trials were calculated to characterise the level of imitation in a pair. T-tests were used to determine whether these values were greater than zero and if they differed from each other.

\section{Results}

Our first analysis shows that Followers tended to imitate the Leader's trajectory with high fidelity, despite not being explicitly asked to do so (Figure 3A). A one-sample ttest showed a statistically significant correlation between the height reached by the Follower and the height reached by the Leader $[\mathrm{N}=30, \mathrm{M}=0.38$, Std. Dev. $=0.46, \mathrm{p}<0.001]$.

Our second analysis tests the core experimental question: do participants imitate more when they know they are being watched, compared to when they are not watched? Figure $3 \mathrm{C}$ shows the peak heights reached by the Leader and the Follower for one sample dyad. Across all participants, we compared the correlation between the peak heights reached by the Leader and the Follower in trials where the Leader was watching the Follower make their movements and the trials where the Leader was not watching. A paired-sample t-test $[\mathrm{N}=30]$ showed that these $\mathrm{R}$-values were higher when the Leader was watching $[\mathrm{M}=0.48$, Std. Dev. $=0.45]$ than when the Leader was not watching $[\mathrm{M}=0.32$, Std. Dev $=0.55]$ and that this effect was significant $[t(29)=2.84, p=0.008]$.

This supports our hypothesis that participants will imitate to a greater extent when they know they are being watched by their partner when compared with a situation where they know their partner cannot see them. Both these results are similar to results previously seen in the pilot experiment (see Supplementary Materials). Further exploratory analyses of movement timing and multi-level regressions are detailed in the Supplementary Materials. 
A

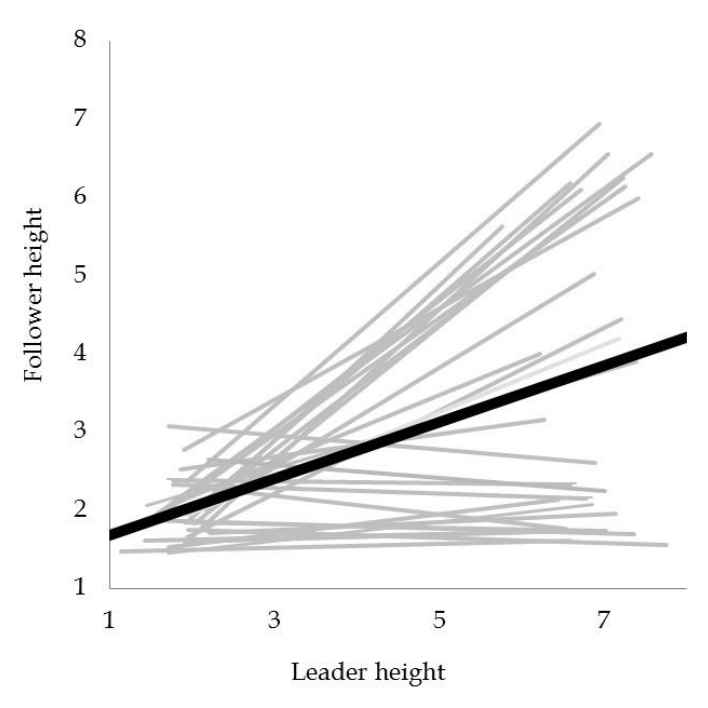

B

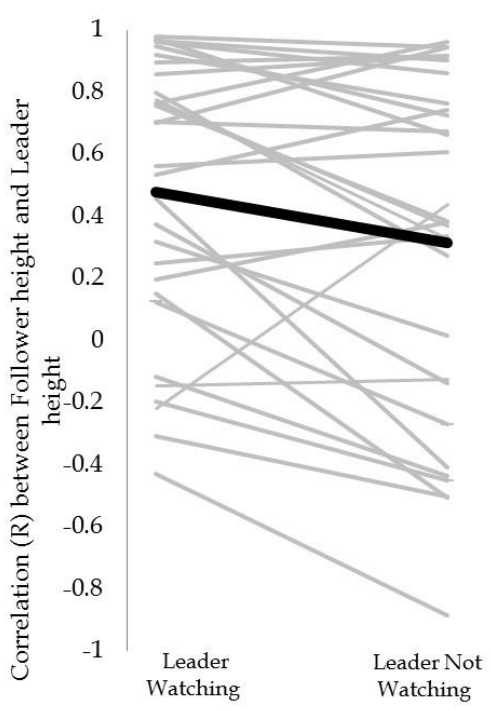

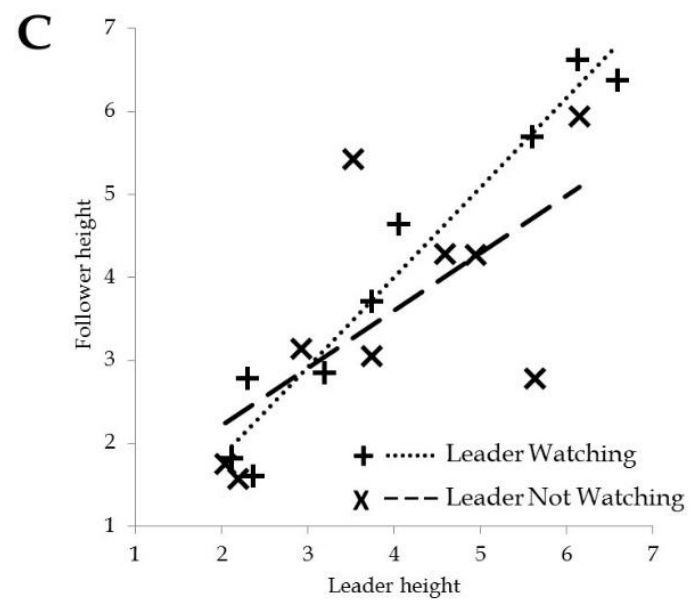

Figure 3. A. Overall imitation pattern Line of best-fit for the correlation between Leader heights and Follower heights for each dyad (grey lines) and the group as a whole (heavy black line). B. Correlations: The $\mathrm{R}$ values representing the correlation between the Leader and Follower heights are shown for each dyad and for the whole group (heavy black line) for trials where the Leader watches the Follower make their movements and trials where the Leader does not watch. C. Results for one sample dyad: The heights reached by the Leader and Follower in one dyad (\#20) and respective trendlines across both the Watched and Unwatched conditions.

The results also suggest that there are sizeable individual differences in Followers' propensity to imitate (Figure 3A). Some Followers imitated Leaders with great fidelity, while others did not. An exploratory analysis linking imitative behaviour to traits measured 
via our questionnaires, namely anxiety, rapport or autistic traits did not suggest any relationships between these self-reported traits and imitative behaviour ${ }^{3}$.

\section{Discussion}

This experiment aimed to test the social-signalling hypothesis of imitation that posits that one of the reasons that humans imitate others is to send a social signal. In this study we found clear evidence that adults imitate with greater fidelity when they know they are being watched by an interaction partner.

The current study advances previous work on imitation in several important ways. First, by using two naïve participants (rather than having a confederate or experimenter demonstrate the action sequence) and pre-registering the analyses, we can be confident that our results do not reflect experimenter biases. Second, using augmented-reality allowed for precise capture of motion kinematics while maintaining good ecological validity. Third, the 'feeling of being watched' was manipulated at an abstract level by voice signals instructing the Leader to open or close their eyes. This allows us to rule out several alternative interpretations of differences in imitative behaviour being due to arousal from direct gaze (Senju \& Johnson, 2009), due to social facilitation (Zajonc, 1965), or due to varying levels of anxiety or attention (Heyes, 2017). In our study both participants stood side-by-side throughout the experiment and therefore differences in eye-gaze and social-facilitation cannot explain the present results. Participants were asked to move quickly and accurately, implying that a straight trajectory was more efficient than using a curved one. Further, if participants felt more anxious during trials when they know they are being watched (Zajonc, 1965), we would expect them to move faster, straighter and lower. Yet, participants actual movements were higher and did not differ in speed. Finally, since the demonstration phases were identical in both the watched and unwatched conditions there cannot be systematic differences in attention during the demonstration phase, allowing us to rule out attentional explanations.

Overall, our experimental design suggests that the 'being watched' effect does not arise from differences in arousal, social facilitation, anxiety or attention. The remaining explanation is that participants imitate with greater fidelity in order to send a signal to their

${ }^{3}$ It is important to note that this does not mean there is no relationship. Our questionnaires were exploratory in nature and we did not set out to test individual differences. We expect that a much larger study may be more suited to examining these in detail. 
interaction partner. That is, these results support the claim that imitation can serve as a social signal (Farmer et al., 2018) and suggests that this signal is enhanced when senders know the recipient can receive it. This is compatible with the STORM model which posits that basic mechanisms for observing and performing actions can be modulated according to the scope and need to strengthen a social connection (Wang \& Hamilton, 2012). The current data is also consistent with earlier work on emotion mimicry (Bavelas et al., 1986) and studies using video stimuli (Wang, Newport, \& Hamilton, 2011). Note that the claim that imitation can be a social signal does not rule out the possibility that, in other contexts, imitation can also be used for social learning (Lyons, Damrosch, Lin, Macris, \& Keil, 2011), as many functions can coincide in this behaviour (Over \& Carpenter, 2012).

There are also some limitations to our results. We cannot determine if Followers became consciously aware of the Leader's unusual trajectories at some point prior to being explicitly told about the trajectories (in the final phase) ${ }^{4}$. This study, therefore, does not distinguish between conscious and unconscious copying. Future studies could measure when (if ever) Followers become aware of the unusual trajectories and test if awareness modulates imitation fidelity. Second, this study set social imitation of kinematics within the context of a block-order learning task; a potential manipulation for future experiments would be to generate a paradigm without a learning objective, such as a task involving only natural conversation.

Our study also generates several possible directions for future research. First, if imitation is being used as a social signal, what message is being sent? Previous work has suggested that imitation signals a desire to affiliate (Chartrand \& Lakin, 2012) but positive effects of being imitated are not always seen (Hale \& Hamilton, 2016a; Verberne, Ham, \& Midden, 2015). Kinematic patterns can also signal informative intentions (McEllin, Sebanz, \& Knoblich, 2018) or confidence (Patel, Fleming, \& Kilner, 2012) which could be important here. It would also be interesting to understand the neural mechanisms of imitation as a social signal. The STORM model suggests that the interaction between gaze and imitation arises due to influence of medial prefrontal cortex on mirror neuron regions (Wang \& Hamilton, 2013; Wang, Ramsey, \& Hamilton, 2011). Combining this paradigm with brain imaging techniques such as functional near-infrared spectroscopy (fNIRS) (Pinti et al., 2018), will allow us to test the brain mechanisms involved while preserving the ecological validity

${ }^{4}$ We could not explicitly ask about this since we did not want to prime participants with questions about trajectories until the end of the experiment. 
of the dyadic interaction. Finally, our results suggest that some Followers imitated with greater fidelity than others, although we did not find links between the self-reported traits and propensity to imitate. Examining these individual differences could also be a productive avenue for further study.

\section{Conclusions}

We hypothesised that imitation functions as a social signal and would be modulated in line with its expected communicative capacity. A preregistered study of 30 pairs of naïve participants shows that participants tend to imitate the causally-irrelevant kinematic features of their partner's movements, and imitate more when they knew their partner could see them. This provides evidence for top-down social modulation of imitation (Wang \& Hamilton, 2012) and for the use of imitation behaviour as a social signal to others.

\section{Acknowledgements}

AH is supported by ERC grant 313398-INTERACT. SKB is supported by an ESRC PhD studentship.

\section{Author Contributions}

Both authors contributed to the study concept and design. Testing and data collection were performed by SKB. SKB performed the data analysis and interpretation under the supervision of AH. SKB drafted the manuscript and AH provided critical revisions. Both authors approved the final version of the manuscript for submission.

\section{Open practices statement}

This study was preregistered at https://osf.io/ezj8g/. Anonymised data will be available on OSF when the paper is published.

\section{References}

Bavelas, J., Black, A., Lemery, C. R., \& Mullett, J. (1986). “I show how you feel”: Motor mimicry as a communicative act. Journal of Personality and Social Psychology, 50(2), 322-329. http://doi.org/10.1037/0022-3514.50.2.322

Bond, C. F. (1982). Social facilitation: A self-presentational view. Journal of Personality and Social Psychology, 42(6), 1042-1050. http:/ / doi.org/10.1037/0022-3514.42.6.1042

Chartrand, T. L., \& Lakin, J. L. (2012). The Antecedents and Consequences of Human Behavioral Mimicry. Annual Review of Psychology, 64(1), 285-308. http://doi.org/10.1146/annurev-psych-113011-143754

Diyanni, C., Nini, D., \& Rheel, W. (2011). Looking good versus doing good: which factors take 
precedence when children learn about new tools? Journal of Experimental Child Psychology, 110(4), 575-91. http:// doi.org/10.1016/j.jecp.2011.06.002

Farmer, H., Ciaunica, A., \& Hamilton, A. F. de C. (2018). The functions of imitative behaviour in humans. Mind E Language. http:/ / doi.org/10.1111/mila.12189

Faul, F., Erdfelder, E., Lang, A.-G., \& Buchner, A. (2007). G*Power 3: a flexible statistical power analysis program for the social, behavioral, and biomedical sciences. Behavior Research Methods, 39(2), 175-91. http:// doi.org/10.3758/BF03193146

Flynn, E., \& Smith, K. (2012). Investigating the mechanisms of cultural acquistion: How pervasive is overimitation in adults? Social Psychology, 43(4), 185-195. http://doi.org/http://dx.doi.org/10.1037/xge0000076

Gilder, T. S. E., \& Heerey, E. A. (2018). The Role of Experimenter Belief in Social Priming. Psychological Science, 095679761773712. http:// doi.org/10.1177/0956797617737128

Hale, J., \& Hamilton, A. F. de C. (2016a). Cognitive mechanisms for responding to mimicry from others. Neuroscience and Biobehavioral Reviews, 63. http:/ / doi.org/10.1016/j.neubiorev.2016.02.006

Hale, J., \& Hamilton, A. F. de C. (2016b). Testing the relationship between mimicry, trust and rapport in virtual reality conversations. Scientific Reports, 6, 35295. http://doi.org/10.1038/srep35295

Hamilton, A. F. D. C. (2015). Cognitive underpinnings of social interaction. The Quarterly $\begin{array}{llll}\text { Journal of Experimental } & \text { Psychology, 68(3), }\end{array}$ http://doi.org/10.1080/17470218.2014.973424

Heyes, C. (2015). Animal mindreading: what's the problem? Psychonomic Bulletin E Review, 22(2), 313-327. http:/ / doi.org/10.3758/s13423-014-0704-4

Heyes, C. (2017). When does social learning become cultural learning? Developmental Science, 20(2), e12350. http:/ / doi.org/10.1111/desc.12350

Kuhlen, A. K., \& Brennan, S. E. (2013). Language in dialogue: when confederates might be hazardous to your data. Psychonomic Bulletin $\mathcal{E}$ Review, 20(1), 54-72. http:/ / doi.org/10.3758/s13423-012-0341-8

Lakin, J. L., \& Chartrand, T. L. (2003). Using nonconscious behavioral mimicry to create affiliation and rapport. Psychological Science, 14(4), 334-339. http://doi.org/10.1111/1467-9280.14481

Lakin, J. L., Jefferis, V. E., Cheng, C. M., \& Chartrand, T. L. (2003). THE CHAMELEON EFFECT AS SOCIAL GLUE : EVIDENCE FOR THE EVOLUTIONARY SIGNIFICANCE OF NONCONSCIOUS MIMICRY. Journal of Nonverbal Behavior, 27(3), 145-162. http:// doi.org/10.1023/1025389814290

Lyons, D. E., Damrosch, D. H., Lin, J. K., Macris, D. M., \& Keil, F. C. (2011). The scope and limits of overimitation in the transmission of artefact culture. Philosophical Transactions of the Royal Society of London. Series B, Biological Sciences, 366(1567), 1158-1167. http://doi.org/10.1098/rstb.2010.0335

Lyons, D. E., Young, A. G., \& Keil, F. C. (2007). The hidden structure of overimitation. 
Proceedings of the National Academy of Sciences of the United States of America, 104(50), 197516. http://doi.org/10.1073/pnas.0704452104

McEllin, L., Sebanz, N., \& Knoblich, G. (2018). Identifying others' informative intentions from movement kinematics. Cognition, 180, 246-258. http:/ / doi.org/10.1016/J.COGNITION.2018.08.001

Nadel, J. (2002). Imitation and imitation recognition : Functional use in preverbal infants and nonverbal children with autism. The Imitative Mind Development Evolution and Brain, 4262. http:// doi.org/10.1017/CBO9780511489969.003

Nielsen, M., \& Blank, C. (2011). Imitation in Young Children: When Who Gets Copied Is More Important Than What Gets Copied. Developmental Psychology, 47(4), 1050-1053. http://doi.org/10.1037/a0023866

Oliver, D., Tachtsidis, I., \& Hamilton, A. F. de C. (2017). The role of parietal cortex in overimitation: a study with fNIRS. Social Neuroscience, 1-12. http:/ / doi.org/10.1080/17470919.2017.1285812

Over, H., \& Carpenter, M. (2012). Putting the social into social learning: explaining both selectivity and fidelity in children's copying behavior. Journal of Comparative Psychology (Washington, D.C. : 1983), 126(2), 182-92. http:// doi.org/10.1037/a0024555

Over, H., \& Carpenter, M. (2013). The Social Side of Imitation. Child Development Perspectives, 7(1), 6-11. http://doi.org/10.1111/cdep.12006

Patel, D., Fleming, S. M., \& Kilner, J. (2012). Inferring subjective states through the observation of actions. Proceedings. Biological Sciences / The Royal Society, 279(October), 4853-60. http://doi.org/10.1098/rspb.2012.1847

Pinti, P., Tachtsidis, I., Hamilton, A., Hirsch, J., Aichelburg, C., Gilbert, S., \& Burgess, P. W. (2018). The present and future use of functional near-infrared spectroscopy (fNIRS) for cognitive neuroscience. Annals of the New York Academy of Sciences. http://doi.org/10.1111/nyas.13948

Risko, E. F., Laidlaw, K. E. W., Freeth, M., Foulsham, T., \& Kingstone, A. (2012). Social attention with real versus reel stimuli: toward an empirical approach to concerns about ecological validity. Frontiers in Human Neuroscience, 6(May), 1-11. http:// doi.org/10.3389/fnhum.2012.00143

Senju, A., \& Johnson, M. H. (2009). The eye contact effect: mechanisms and development. Trends in Cognitive Sciences, 13(3), 127-34. http:/ / doi.org/10.1016/j.tics.2008.11.009

Teufel, C., Fletcher, P. C., \& Davis, G. (2010). Seeing other minds: attributed mental states influence perception. Trends in Cognitive Sciences, 1-7. http:// doi.org/10.1016/j.tics.2010.05.005

Uzgiris, I. (1981). Two Functions of Imitation During Infancy. International Journal of Behavioral Development, 4(1), 1-12. http:/ / doi.org/10.1177/016502548100400101

Verberne, F. M. F., Ham, J., \& Midden, C. J. H. (2015). Trusting a Virtual Driver That Looks, Acts, and Thinks Like You. Human Factors: The Journal of the Human Factors and Ergonomics Society, 57(5), 895-909. http:/ / doi.org/10.1177/0018720815580749 
Wang, Y., \& Hamilton, A. F. D. C. (2012). Social top-down response modulation (STORM): a model of the control of mimicry in social interaction. Frontiers in Human Neuroscience, 6(June), 153. http://doi.org/10.3389/fnhum.2012.00153

Wang, Y., \& Hamilton, A. F. de C. (2013). Understanding the Role of the "Self" in the Social Priming of Mimicry. PLoS ONE, 8(4). http:// doi.org/10.1371/journal.pone.0060249

Wang, Y., Newport, R., \& Hamilton, A. F. de C. (2011). Eye contact enhances mimicry of intransitive hand movements. Biology Letters, 7(1), 7-10. http://doi.org/10.1098/rsbl.2010.0279

Wang, Y., Ramsey, R., \& Hamilton, A. F. de C. (2011). The control of mimicry by eye contact is mediated by medial prefrontal cortex. The Journal of Neuroscience: The Official Journal of the Society for Neuroscience, 31(33), 12001-10. http:/ / doi.org/10.1523/JNEUROSCI.084511.2011

Whiten, A., Allan, G., Devlin, S., Kseib, N., Raw, N., \& McGuigan, N. (2016). Social learning in the real-world: "Over-imitation" occurs in both children and adults unaware of participation in an experiment and independently of social interaction. PLoS ONE, 11(7), 1-14. http://doi.org/10.1371/journal.pone.0159920

Zajonc, R. B. (1965). SOCIAL FACILITATION. Science (New York, N.Y.), 149(3681), 269-74. 


\title{
Adults imitate to send a social signal
}

\author{
Sujatha Krishnan-Barman and Antonia F de C Hamilton
}

\section{Supplementary Materials}

The main paper summarises the methods used to conduct the preregistered study and analyse the data. The Supplementary Materials provide further details on the methods in order to aid any researchers wishing to replicate or extend our results.

The Supplementary Materials also detail certain exploratory analyses conducted on the data, which are noted in the main text but elaborated on here.

\section{Pilot study}

\subsection{Methods}

22 dyads were tested in the pilot study. In 8 of these dyads, the role of leader was taken by a confederate because one participants failed to arrive for the booked slot. Procedures were identical to the reported full study, except that trials had 3 or 5 blocks per trial (not 3 or 4).

\section{$10.2 \quad$ Results}

Our first analysis shows that Followers tended to imitate the Leader's trajectory with high fidelity, despite not being explicitly asked to do so. A one-sample t-test showed a statistically significant correlation (R_overall) between the height reached by the Follower and the height reached by the Leader $[\mathrm{N}=22, \mathrm{M}=0.30$, Std. Dev. $=0.43, \mathrm{p}=0.04]$.

Our second analysis tests the core experimental question: do participants imitate more when they know they are being watched, compared to when they are not watched? Across all participants, we compared the correlation between the peak heights reached by the Leader 
and the Follower in trials where the Leader was watching the Follower make their movements and the trials where the Leader was not watching. A paired-sample t-test [ $\mathrm{N}=22]$ showed that these R-values were higher when the Leader was watching $[\mathrm{M}=0.43$, Std. Dev. $=0.36]$ than when the Leader was not watching $[\mathrm{M}=0.19$, Std. Dev $=0.55]$ and that this effect was significant $[\mathrm{t}(21)=2.96, \mathrm{p}=0.007]$.

\section{Details on Materials and Methods}

\subsection{Sample size rationale}

The main study is a pre-registered replication of our pilot study (https://osf.io/ezj8g/) and all power calculations were based on our pilot study. We carried out a power analysis in G*Power (Faul, Erdfelder, Lang, \& Buchner, 2007) to determine the minimum number of participants needed to detect a within-participant effect of social context on imitation. Analysis of the pilot data showed that social context (being watched) significantly increased imitation with an effect size $d z=0.63$. Using this estimate, the analysis suggested that 29 dyads would be sufficient to detect an effect with a power of 0.9. We therefore planned to collect data from 30 valid dyads.

\subsection{Stopping rule}

Given that we had to exclude dyads from the final analysis based on the criteria below, we planned to continue testing until we collect data from 30 valid dyads. Based on these criteria we eventually needed to test 40 dyads in order to obtain 30 valid dyads.

\subsection{Exclusion criteria}

Dyads were excluded if they failed to follow the instructions:

(i). in the Experimental phase, if the Leader fails to copy the trajectory height of the computer demonstration (with a Pearson's correlation coefficient value of at least 0.5 or higher) once the Leader had been explicitly instructed to do so; or 
(ii). in the Final phase, once both the Leader and the Follower were instructed to explicitly follow the heights of the trajectories, if the Follower then failed to copy the Leader with high fidelity (with a Pearson's correlation coefficient value of 0.5 or higher).

(iii). The Leader mentioned that they had been given a secret instruction to follow the trajectory heights to the Follower prior to being allowed to do so.

(iv). The Follower ignored the instruction to only discuss the order of the blocks and asked the Leader explicitly about the path or trajectories they were taking.

(v). Either the Leader or the Follower failed to follow the instructions to close their eyes at various points.

We also excluded dyads if:

(i). Participants were familiar with each other prior to the study

(ii). Data was not recorded owing to either equipment failure or a failure in the task software

(iii). Motion sensors moved or fell off during the study

(iv). The study was stopped midway for any other reason, such as participant fatigue

\subsection{Additional measures}

Following the block-moving task, participants individually completed the following questionnaires: (i) Rapport questionnaire: Six-item survey of their feelings of rapport towards each other (see Section 4: Questionnaires), (ii) Autism Spectrum Quotient (AQ) questionnaire (Baron-Cohen, Wheelwright, Skinner, Martin, \& Clubley, 2001) which measures the extent of autistic traits in adults, (iii) Interaction Anxiousness Scale (Leary, 1983) which measures social anxiety, and (iv) Rosenberg Self-Esteem Scale (Rosenberg, 1965) which measures self-esteem. Participants also completed a written debrief in which they were asked what they thought the purpose of the experiment was, and whether they noticed the differences in the trajectory heights prior to being explicitly told about them. Further, they were asked to self-report their ethnicity and their level of familiarity with their partner and the experimenter. In addition to tracking motion using a motion-capture system, we 
also recorded videos of the participants performing the task. This allows us to verify that they kept their eyes closed and refrained from talking when required to do so, ensuring that the social manipulation worked.

\subsection{Phases of the experiment}

This table summarises the different phases of the experiment and the number of trials included in each phase.

\begin{tabular}{lccccccr} 
& $\begin{array}{c}\text { Explicitly instructed } \\
\text { to copy trajectory of } \\
\text { demonstration? }\end{array}$ & $\begin{array}{c}\text { Follower's eyes } \\
\text { during } \\
\text { Phase }\end{array}$ & \begin{tabular}{c} 
Leader \\
\cline { 2 - 5 } Follower
\end{tabular} & $\begin{array}{c}\text { Leader's eyes } \\
\text { during } \\
\text { follower's } \\
\text { movement }\end{array}$ & $\begin{array}{c}\text { Trajectory } \\
\text { heights }\end{array}$ & $\begin{array}{r}\text { Total } \\
\text { number } \\
\text { of trials }\end{array}$ \\
\hline Familiarisation & No & No & Close & Open & Low / Med / High & 3 \\
\hline Full & Yes & No & Close & Open / Close & Low / Med / High & 18 \\
\hline Final & Yes & Yes & Close & Open / Close & Low / Med / High & 6 \\
\hline
\end{tabular}

\subsection{Data analysis}

In each trial the Leader and the Follower each made 3 or 4 movements involving picking up a block from the source table and placing it on the target location. The trajectories were recorded using the motion-capture software and the highest height reached by the participant for each movement was computed. The position of the blocks on the table was shuffled between the Leader's turn and the Follower's turn in each trial, so we used the overall maximum height reached by each participant (across all 3 or 4 movements) as the maximum height recorded for that trial.

The dependent variable of interest was the correlation coefficient $(R)$ between the maximum height reached by the Follower in each trial and the maximum height reached by the Leader in their demonstration in that trial. That is, we were interested in seeing whether the Follower copied the causally-irrelevant aspects (trajectory height) of the Leader's movements in addition to the causally-relevant aspects (block order). We calculated the $\mathrm{R}$ value for each pair of participants in the 'eyes open' and 'eyes closed' conditions and used ttests to determine if these values were greater than zero and if they differed from each other. 


\section{Exploratory analysis on the preregistered study}

\subsection{Multi-level regression analysis}

In addition to the analysis in the main text, we performed a more detailed exploration of which factors predict the height of the follower's action, using a multi-level regression analysis. The factors considered were

a. the dyad number

b. the maximum height reached by the Leader

c. the number of pieces to be moved in each trial

d. the trial number

e. whether the Leader watched the Follower make their movements or if this was unwatched

f. and a variable to capture the interaction effect between the height reached by the Leader and whether the Follower was watched or unwatched

\section{Pilot study}

For the pilot study, we found that, using the Enter method, a model incorporating the dyad number (Dyad_number), the height reached by the leader (L_Height), and whether the leader had their eyes open or closed during the follower's turn (L_Open_Close) accounted for 10.9\% of the variation in the height reached by the follower (F_Height) $[F(3,392)=15.97 ; p<0.001]$. Introducing the interaction effect between the height reached by the leader and whether the leader's eyes were subsequently open during the follower's turn (L_Height_x_L_Open_Close) explained an additional $1 \%$ of variation in F_Height and this R2 change was significant $[\Delta \mathrm{F}(1,391)=4.36 ; \mathrm{p}=0.038]$. In the second stage there was no main effect of dyad number (Dyad_number) or whether the leader had their eyes open or closed during the follower's turn (L_Open_Close) on F_Height. However, both the height reached by the leader (L_Height) $[\mathrm{t}(391)=6.4 ; \mathrm{p}<0.001]$ and the interaction effect (L_Height_x_Watched_Unwatched) $[\mathrm{t}(391)=$ -2.09; $\mathrm{p}=0.038$ ] did significantly predict the height reached by the follower (F_Height). 


\section{Main study}

In the pre-registered study, our results varied a little. Using the Enter method it was found that a model incorporating these variables accounted for $19 \%$ of the variation in the height reached by the Follower $[F(6,533)=21.30, p<0.001]$. This model suggests that the height reached by Leader was predictive of height subsequently reached by Follower $[t(535)=$ 10.03; $\mathrm{p}<0.001]$. A main effect of trial number $[\mathrm{t}(535)=2.57 ; \mathrm{p}=0.011]$ suggests imitation increased as the experiment went on, while the main effect of dyad number $[\mathrm{t}(535)=3.65$; $p<0.001]$ is indicative of individual differences between dyads. There was no main effect of the number of pieces to be moved and whether the Leader watched the Follower make their movements. The interaction between the Leader's height and whether the Follower was watched while making their movement was not significant $[\mathrm{t}(535)=0.707 ; \mathrm{p}=0.48]$.

\subsection{Exploratory analysis of movement duration}

In addition to the analyses above, we conducted an exploratory analysis to test if the duration of the Leader's movements affected the duration of the follower's movements or was changed by being watched. Throughout the task, participants were encouraged to move quickly because the score they received at the end of each trial was partly based on movement speed.

For each trial of each participant in the dyad, we segmented the recorded movement into the 3 or 4 block movements and calculated the duration of each from first lifting the block from table 1 to placing it on table 2. We took the median duration as representative of that trial, and used the Leader and Follower median durations for further analysis. We find that, in both watched and unwatched trials, the median Follower duration correlated with the median Leader duration, as measured by the r-value for the correlation within each dyad. That means that the mean $r$ value over all dyads was significantly greater than zero for both watched $(t=3.28, d f=29, p=0.0027)$ and unwatched $(t=2.95, d f=29, p=0.0061)$ trials. However, the correlation was not significantly greater on watched trials compared to unwatched trials $(t=0.40, d f=29, p=0.70)$, which means that Followers did not imitate the duration of movements more when they were being watched. 


\subsection{Exploratory analysis of questionnaire data}

Our questionnaires showed that none of the participants were outliers on the traits measured in our questionnaires, namely anxiety, rapport between pairs of participants and autistic traits. Our exploratory analysis of individual differences did not suggest any relationships between the self-reported traits and imitative behaviour. While this could potentially be an area to study in the future, there is no clear path forward based solely on our self-report questionnaire at the moment. Assuming the effect size is medium, for a significance test of correlation coefficient $r$ with a power of 0.80 and $\alpha=.05$ Cohen (1992) suggests that a sample size of 85 would be required.

\section{Questionnaires}

\subsection{Rapport questionnaire}

This is a six-item questionnaire used to measure the participant's feeling of rapport towards each other, with participants rating their degree of agreement or disagreement with each statement on a seven-point Likert scale.

Please read each statement carefully, and rate how strongly you agree or disagree with the statement.

1. The interaction with my partner was very smooth

$$
\begin{array}{lllllllll}
\text { Strongly disagree } & 1 & 2 & 3 & 4 & 5 & 6 & 7 \text { Strongly agree }
\end{array}
$$

2. I felt rapport with my partner

$$
\begin{array}{lllllllll}
\text { Strongly disagree } & 1 & 2 & 3 & 4 & 5 & 6 & 7 \text { Strongly agree }
\end{array}
$$

3. I felt that the communication flow with my partner was easy

$$
\begin{array}{lllllllll}
\text { Strongly disagree } & 1 & 2 & 3 & 4 & 5 & 6 & 7 \text { Strongly agree }
\end{array}
$$


4. I felt very comfortable during the interaction

$$
\begin{array}{lllllllll}
\text { Strongly disagree } & 1 & 2 & 3 & 4 & 5 & 6 & 7 \text { Strongly agree }
\end{array}
$$

5. During the interaction I found it easy to express what I wanted to say

$$
\begin{array}{lllllllll}
\text { Strongly disagree } & 1 & 2 & 3 & 4 & 5 & 6 & 7 \text { Strongly agree }
\end{array}
$$

6. I felt that my partner could easily understand what I was thinking

$$
\begin{array}{lllllllll}
\text { Strongly disagree } & 1 & 2 & 3 & 4 & 5 & 6 & 7 \text { Strongly agree }
\end{array}
$$

\subsection{Familiarity questionnaire}

How well did you know your teammate before the game?

- Unfamiliar (did not know him/her at all)

- Somewhat familiar (casual acquaintance)

- Familiar (friend)

- Very familiar (long-time friend, roommate, partner, family member etc)

If you knew your teammate before the game, how long have you known each other?

[Text box]

If you knew your teammate before the game, how often have you met in the past week?

- Not met in the past week

- Met once

- Met more than once but not everyday

- Met everyday

How well did you know the experimenter before the game?

- Unfamiliar (did not know him/her at all)

- Somewhat familiar (casual acquaintance) 
- Familiar (friend)

- Very familiar (long-time friend, roommate, partner, family member etc)

\section{References}

Cohen, J. (1992). A power primer. Psychological Bulletin, 112(1), 155-159. http:/ / doi.org/10.1037/0033-2909.112.1.155

Faul, F., Erdfelder, E., Lang, A.-G., \& Buchner, A. (2007). G*Power 3: a flexible statistical power analysis program for the social, behavioral, and biomedical sciences. Behavior Research Methods, 39(2), 175-91. http:// doi.org/10.3758/BF03193146 